\title{
Tracking priors and their replacement: Mental dynamics of decision making in the number-line task ${ }^{\circ}$
}

\author{
Dror Dotan ${ }^{1,2}$ and Stanislas Dehaene $e^{1,3}$ \\ ${ }^{1}$ CEA-INSERM Cognitive Neuroimaging Unit, France. ${ }^{2}$ Mathematical Thinking Lab, School of Education and \\ School of Neuroscience, Tel Aviv University. ${ }^{3}$ Collège de France.
}

\begin{abstract}
Several theories of decision making assume that optimal decisions are reached by computing a prior distribution over possible responses, and then updating it according to the evidence received. We show how this prior replacement, with its two processing stages, can be captured with a simple behavioral method: tracking the finger movement as participants point to a response location. On each trial, participants saw a number and pointed to its location on a number line. In two experiments, we manipulated either the prior, via the distribution of target numbers, or the initial finger direction, via explicit instruction. In both experiments, when a trial started the participants pointed toward the instructed direction, and in the last part of the trial they pointed toward the target. Critically, between these two stages there was a third, interim stage in which the participants pointed toward the prior before deviating toward the target. Transient pointing toward the prior was observed even when it induced a brief deviation away from the target. This pattern fits a model wherein decisions are first driven by prior knowledge, followed by the accumulation of trial-specific evidence. We propose that the number-to-position mapping task with finger tracking is a powerful paradigm to investigate fine-grained aspects of priors in a simple decision-making scenario.
\end{abstract}

\section{Introduction}

Hundreds of times a day we need to choose one action among several possible ones. The last two decades have seen major advances in our understanding of the cognitive and neural processes involved in simple decision making, and in our ability to account for simple decisions with formal mathematical models (Glimcher, 2003; Gold \& Shadlen, 2007; Kiani et al., 2014; Ratcliff \& McKoon, 2007). Such models consider that human decisions are close to the normative optimum provided by Bayesian reasoning: they assume that decision-makers maintain a likelihood for each possible decision outcome (Barthelmé \& Mamassian, 2010; Knill \& Pouget, 2004; Smith \& Ratcliff, 2004), and continuously update these likelihoods by accumulating evidence arising from the stimulus, until a decision criterion is met (Gold \& Shadlen, 2002; Kiani et al., 2008; Lo \& Wang, 2006; Ratcliff \& Rouder, 1998; Roitman \&

\footnotetext{
'We thank Florent Meyniel and Maxime Maheu for their comments on earlier versions of the manuscript, and Ricardo Tarrasch for the statistical advice. This research was supported by INSERM, CEA, Collège de France, Universite Paris-Sud, the Azrieli Foundation (Azrieli Fellowship to Dror Dotan), and the Jacobs Foundation (grant no. 2019-1320-05, Dotan). The research was a part of the doctoral dissertation of Dror Dotan in Tel Aviv University, under the supervision of Naama Friedmann and Stanislas Dehaene.
} 
Shadlen, 2002). In accord with such models, the activity of neurons in prefrontal and parietal cortices appears to build over time, with a slope monotonically related to the available evidence, up to a threshold level, as expected under the hypothesis that they encode the relative likelihoods of the decision outcomes (Kiani \& Shadlen, 2009; O'Connell et al., 2012; Roitman \& Shadlen, 2002). Furthermore, stronger activity, presumably reflecting a sharper distribution of likelihoods, results in higher confidence in the decision (Kiani \& Shadlen, 2009).

One of the pillars of the such theoretical frameworks, and the topic of the present study, is the notion of a prior: even before any evidence is obtained, we presumably entertain a prior distribution of likelihoods, based on our past experience and expectations. This prior is progressively combined with an accumulation of the evidence received on a given trial. Mathematical models with a prior successfully account for behavior in a variety of decision tasks (Kording \& Wolpert, 2004; Stocker \& Simoncelli, 2006). Moreover, participants' responses and brain activity are demonstrably biased by the previous distribution of stimuli in the experiment, in line with the notion that past experience is used to shape the prior for the next trial (Cicchini et al., 2014; Fernandes et al., 2014; Hanks et al., 2011; Kording \& Wolpert, 2004; Summerfield \& de Lange, 2014; Tassinari et al., 2006).

Nevertheless, the evidence in support of this prior replacement model - the idea that a decision can start with a prior, which is then updated based on accumulated evidence - is still limited. Behavioral choices and response times only provide a summary of the total decision period and are largely blind to its dynamics. Neurophysiological studies can reveal the temporal unfolding of decision variables during a trial (Yang \& Shadlen, 2007) (see review in Summerfield \& de Lange, 2014), but they either involve invasive electrophysiology in highly trained animals, or indirect brain measures which are hard to attribute to a specific neural system in human subjects (O'Connell et al., 2012). It would be extremely useful if a purely behavioral measure was available to directly capture, in humans, the order and rate at which priors and evidence accumulation occur on each trial of an instructed decision task.

Here we used a simple behavioral measure that fits the bill: we continuously recorded the participants' finger movement as they took a decision by pointing to a response location, and we analyzed the factors that affect the finger movement in different times during the trial. Previous studies have confirmed that the the pointing direction can change during the trial and reflect the ongoing decision process (Chapman et al., 2010b, 2010a; Dotan, Meyniel, \& Dehaene, 2018; Erb, Moher, Sobel, \& Song, 2016; Finkbeiner \& Friedman, 2011; Pinheiro Chagas, Dotan, Piazza, \& Dehaene, 2017; Santens, Goossens, \& Verguts, 2011; Song \& Nakayama, 2008; see review in Dotan, Pinheiro-Chagas, Al Roumi, \& Dehaene, 2019). We reasoned that the finger movement would reflect a succession of prior followed by evidence accumulation: for a short period of time, the finger movement would be affected by the prior, but not yet the evidence arising from the target; later in the trial, once the target is being processed, the finger should gradually deviate towards it. Corresponding with this idea, several studies examined situations of "cognitive competition" between two response alternatives, and showed how the target gradually overcomes the distracter (e.g., Spivey et al., 2005; see review in Stillman et al., 2018). Here, to 
extend this idea from two response alternatives to full-fledged priors, we used a task with numerous response alternatives.

We used a number-to-position mapping task: on each trial, the participants saw an Arabic number between 0 and 100 and pointed at the corresponding position on a number line (Berteletti et al., 2010; Siegler \& Opfer, 2003) (Fig. 1a). This task requires decision making because it involves multiple sources of uncertainty, arising from multiple stages of processing involved in the task (Dehaene et al., 2008; Dotan \& Dehaene, 2016, 2020; Siegler \& Opfer, 2003). First, the participants identify the digit symbols. Second, they convert the digit symbols into a cognitive representation of the corresponding quantity, whose representation is fuzzy (Cicchini et al., 2014; Dehaene, 1997). Third, they map this quantity to the corresponding location on a "mental number line", and this process too involves uncertainty (Slusser \& Barth, 2017). Last, they perform the motor operation of pointing accordingly to the physical number line that they see on the screen.

In a previous study we proposed a specific ideal-observer Bayesian model of this task (Dotan \& Dehaene, 2016). According to this model, on each trial, the likelihood of each possible response location is initialized to a prior inferred from the distribution of previous target numbers in the experiment. The likelihoods are gradually updated during the trial according to an approximate representation of the target quantity. This model yields two predictions relevant to the notion of a prior: first, the finger should initially move according to the previous distribution of target numbers, and only then towards the target of the present trial. Second, assuming that the prior gets updated by recent information and gradually forgets older information (Abrahamyan et al., 2016; Cicchini et al., 2014), the finger direction during the pointing-by-prior stage should be biased by the target numbers presented recently, with exponentially decreasing influence of trials $\mathrm{N}-1, \mathrm{~N}-2, \mathrm{~N}-3$, etc.

To assess these predictions in the number-to-position task, we examined whether manipulating the distribution of target numbers, which presumably affects the prior, affects the finger's direction. In Experiment 1, the participants were instructed to start each trial with initially pointing to the middle of the line. The distribution of target numbers was biased towards small, average, or large numbers in separate blocks: this should bias the prior (Fig. 1b). Namely, although the prior may be affected by the participants' a-priori assumptions about the task, here the difference between the blocks was not in a-priori assumptions but in the distribution of targets the participants observed in the previous trials in the block. In Experiment 2, as a control, the distribution of target numbers was constantly flat, i.e., we did not manipulate the prior, but the participants' initial finger direction was biased by instructions to initially point to the left, middle or right of the number line in different blocks (Fig. 1c). For both experiments, a trivial prediction is that the finger would first move according to the instructed pointing direction, and then according to the trial-specific target number. Critically, we predicted a third, intermediate stage, during which the finger would move according to the prior. Counter-intuitively, this model predicts that in Experiment 2, on trials where the instructed initial direction points straight at the 
target but the prior differs, subjects should transiently deviate away from the target in order to accommodate the prior.

On top of the theoretical goal, this study also has a methodological goal: to create an experimental paradigm that can be used to examine in detail the representation of priors. As we shall see, the paradigm we introduce here - number-to-position mapping with finger tracking can achieve precisely that, and can serve as a platform for examining the decision making process in real time, including the effect of priors.

\section{Methods}

\subsection{Participants}

Participants were right-handed adults with no reported cognitive disorders, and Hebrew as their native tongue. They were compensated for participation. There were 18 participants in Experiment 1 (mean age $=25 ; 1, \mathrm{SD}=2 ; 4)$ and 24 participants in Experiment 2 (mean age = $26 ; 3, \mathrm{SD}=4 ; 1)$. All participants gave informed consent. The experiment was approved by the Tel Aviv University Ethics Committee.

\subsection{Procedure}

The experiment was run on an iPad touchscreen, placed in landscape orientation (screen resolution: 1024 x 768 pixels, screen refresh rate and finger sampling rate $60 \mathrm{~Hz}$ ). A horizontal number line $(16.2 \mathrm{~cm})$, marked only with 0 and 100 at its ends, occupied the top of the screen throughout the experiment (Fig. 1a). A fixation cross appeared above the middle of the number line upon touching a "start" rectangle, and was replaced by the target number when the finger started moving upwards (when it crossed an imaginary horizontal line just above the start rectangle). The target number was presented in Arial bold white font, with $10 \mathrm{~mm}$ high digits. In the analyses below, the target onset time was defined as time $=0$. The participants then continued moving the finger upwards, dragging it to what they judged to be the corresponding position on the number line, and an arrow indicated the finger landing position. Trials were invalidated whenever the participant lifted the finger in mid-trial, moved backwards, started a trial in an incorrect direction, or moved too slowly: except for an initial grace period of the first $300 \mathrm{~ms}$ of each trial, the finger had to: (1) reach $30 \%$ of the vertical distance to the number line within $1 \mathrm{~s}$ from target onset (with linear interpolation), and the number line itself within $2 \mathrm{~s}$; (2) maintain an instantaneous speed of at least $6 \mathrm{~mm} / \mathrm{s}$. Invalid trials (over the two experiments, $8.36 \%$ of the trials) were excluded from analysis and their targets were presented again later in the experiment. Trials with movement time $<200$ ms were excluded post-hoc.

Note that the participants started moving the finger before the target appeared (in fact, it was the movement initiation that triggered the target onset). This is critical in order to obtain trajectory information right from the start of the trial, including right after the target onset time (Dotan \& Dehaene, 2016; Scherbaum \& Kieslich, 2017).

Each experiment included 3 blocks, administered in random order, with 202 trials per block.

In Experiment 1, unknown to the participants, each condition (block) had different target 
distribution (Fig. 1b): flat (equal probability for all targets); biased to large numbers (the targets 0-33 appeared once, 34-66 appeared twice, and 67-100 appeared 3 times); or biased to small numbers (a mirror symmetry of the large-number condition distribution). In each condition, the 202 experimental trials were preceded by 21 calibration trials, in which the target numbers were selected (with flat distribution) from a biased range - 0-42 in the block biased to small numbers, 58-100 in the block biased to large numbers, or from the whole range 0-100 in the flat block. Calibration trials were aimed to reset the prior bias according to the experimental condition and to override any potential prior from previous blocks. They were administered as part of the block, without informing the participants about their existence, and were not analyzed.

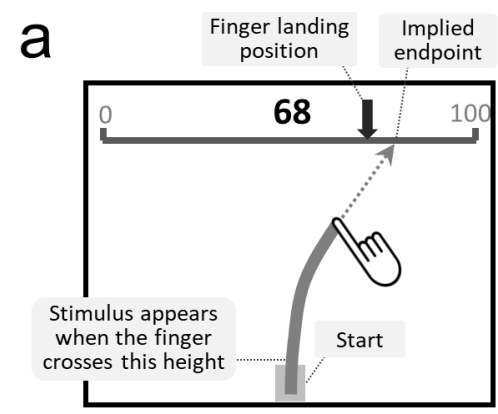

b

Experiment 1: Target distribution per block
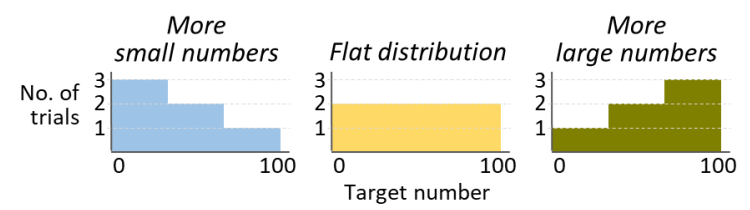

C Experiment 2: Screen layout per block

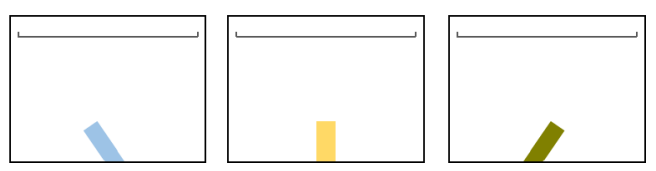

Fig. 1. Logic of the experiments dissociating initial finger direction from prior. (a) Task and screen layout: when participants started to move their finger upward, a 2-digit number target appeared, and subjects pointed to its location on a horizontal number line that extended from 0 to 100. (b) Experiment 1 manipulated the prior by setting different distribution of target numbers per block. The initial finger direction was straight up in all blocks. (c) Experiment 2 used a fixed prior (flat distribution of target numbers) and manipulated the finger initial direction across blocks.

In Experiment 2, all conditions had a flat distribution of targets, and they differed in the finger initial direction (Fig. 1c): the participants were explicitly instructed to start each trial with movement towards the left end of the line (corresponding to target 0 ; the start rectangle was tilted $30^{\circ}$ to the left), to the middle of the line (corresponding to 50; straight start rectangle), or to its right end (corresponding to 100; start rectangle tilted $30^{\circ}$ rightwards). In both experiments, the finger initial direction was enforced: the finger had to leave the "start" rectangle, which was 
tilted $30^{\circ}, 0^{\circ}$ or $-30^{\circ}$, from its top side; if they did not, the trial failed and was introduced again later in the experiment.

\subsection{Data preprocessing}

The data was preprocessed according to a standard method that we used in several previous studies (Al Roumi et al., 2019; Dotan \& Dehaene, 2013, 2016, 2020; Dotan et al., 2018; Pinheiro-Chagas et al., 2017). The finger position was sampled at $60 \mathrm{~Hz}$, recorded as a sequence of time-stamped $\mathrm{x}$ and y coordinates, and transformed into a fixed sampling rate of $100 \mathrm{~Hz}$ using cubic spline interpolation. The $\mathrm{x}, \mathrm{y}$ coordinates were separately smoothed (Gaussian weighting, $\sigma=20 \mathrm{~ms}$ ). Per time point, we calculated the direction between the finger coordinates at times $t-10 \mathrm{~ms}$ and $t$, and the implied endpoint was defined as the position on the number line that the finger would reach if it kept moving in this direction. Implied endpoints outside the range $[-5,105]$ were considered as -5 or 105 . Implied endpoints for sideways movement (|direction $\mid>80^{\circ}$ ) were excluded from the analysis. The implied endpoint reflects the finger momentary aiming direction, which is a better index of the finger movement than the finger horizontal position: with $\mathrm{x}$ coordinates, any factor that modifies the finger direction could be revealed only after the finger had traveled some distance in the new direction (Dotan \& Dehaene, 2013).

\subsection{Trajectory analysis}

To analyze the trajectories we used multiple regression and examined, in each time point during the trial, the factors that affect the finger movement (Dotan et al., 2019). Within each experiment, all three conditions were analyzed together. One multiple regression was run for each participant and time point in $50 \mathrm{~ms}$ intervals, with the implied endpoints as the dependent variable. If a particular trial was shorter than the analyzed time point, we extrapolated it: the post-trial dependent variable value was defined as the finger landing position on the number line. The predictors were the target number (denoted $\mathrm{N}$ ), the target number of the previous trial (denoted N-1), and the Condition. In Experiment 1, the Condition predictor was the average target number in each block (42.5, 50, or 57.5). In Experiment 2, the Condition predictor was the initial direction $(0,50$, or 100). In Experiment 1, we included as predictors not only the target of the preceding trial $(\mathrm{N}-1)$ but also the targets of four preceding trials $(\mathrm{N}-1$ to $\mathrm{N}-4)$, to account for possible correlation between them and the Condition predictor. Furthermore, in Experiment 1, to avoid statistical biases due to between-condition differences in the number of trials with each target number, from each block we included in the regression analysis only one trial for each target number. We used the target's last occurrence in the block, but the results were replicated also when selecting randomly one trial for each target.

To examine whether a given predictor had a significant group-level effect, a second-level analysis was performed per predictor and time point: the individual participants' regression b values (whether significant or not) were compared to zero using t-test across participants (onetailed $p$ is reported because all regression coefficients are predicted to be positive). 
The required sample size for this t-test was calculated a-priori: assuming a relatively weak and noisy effect (such as the log effect observed in a previous study with this paradigm, Dotan \& Dehaene, 2013: regression $b=0.11, \sigma=0.11$ ), setting alpha at 0.01, aiming at a power of 0.9, and using one-tailed analysis, yielded a minimum of 16 participants. Accordingly, we included 18 participants in Experiment 1 and 24 participants in Experiment 2.

Our Matlab analysis tools, and an experimentation software corresponding to the one we used here, are available on http://trajtracker.com. The raw data is available in http://osf.io/7rvzh.

\section{Results}

\subsection{Experiment 1: Manipulating the prior}

Visual inspection of the trajectories suggested that participants were sensitive to the distribution of target numbers: in the two biased-prior conditions, the trajectories were transiently biased leftwards or rightwards (Fig. 2a), a pattern that was observed even on single trials for some participants (Fig. 2b). To quantify this pattern, the trajectory data was submitted to the regression analysis described in Methods: for each time point in the trial, the implied endpoint was entered as the dependent variable in a multiple regression with the following predictors: the Condition (defined as the average target per block), the target number, and the targets of the 4 previous trials (N-1 to N-4). This analysis revealed a succession of three effects (Fig. 2c): in the beginning of a trial, only the constant factor had a strong effect (blue curve), indicating that the finger aimed towards a relatively constant direction - the middle of the number line. This constant factor immediately started declining and completely disappeared by $450 \mathrm{~ms}$ post stimulus onset. In parallel, the finger movement became governed by the Condition factor (red curve). The Condition factor peaked at $350 \mathrm{~ms}$, and started declining as the finger direction became dominated by the trial-specific target number (green).

The Condition predictor is the average target number in the current block - essentially, the average of a prior. Notably, this factor had a significant effect as early as $50 \mathrm{~ms}$ after the target onset; i.e., the participants were probably sensitive to the condition even before the target appeared, in agreement with the idea that this factor reflects the effect of a prior. We verified that this Condition effect was observed not only in the regressions, but also in the trajectories: at the time when the condition effect was at its peak $(350 \mathrm{~ms})$, the average implied endpoint was 41.0 for the left-bias condition, 47.4 for the unbiased condition, and 53.6 for the right-bias condition.

Because the Condition predictor correlated with the target numbers $(r=.32)$, its effect could have been arguably explained as perseveration from recent trials - e.g., because the motor system may be biased towards recent movement plans (Chapman et al., 2010b). However, the results clearly refute this alternative interpretation: the Condition effect could not be reduced to an effect of the recent target numbers, because it had a significant and strong effect although the regression model also included the targets of the 4 recent trials. 

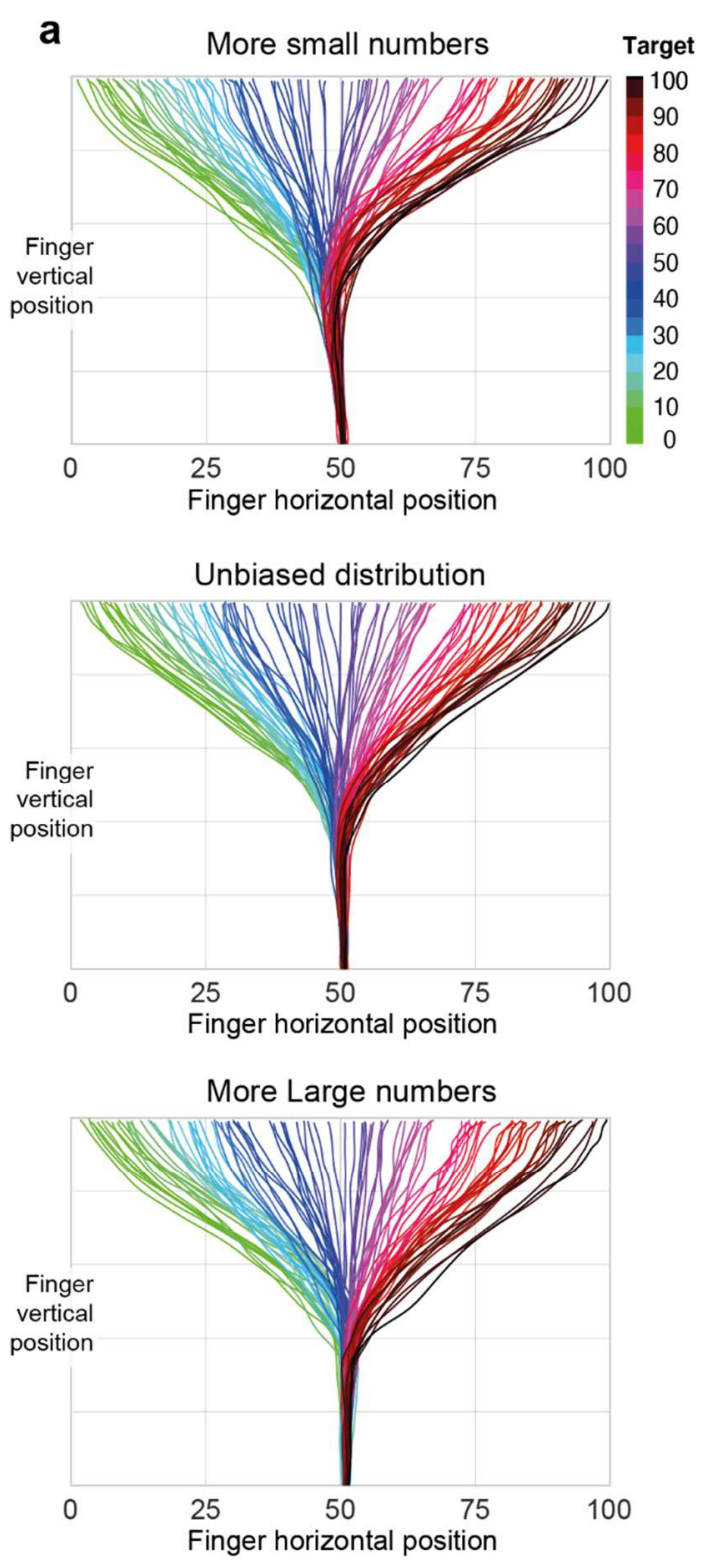

b Sample raw trajectories of one participant

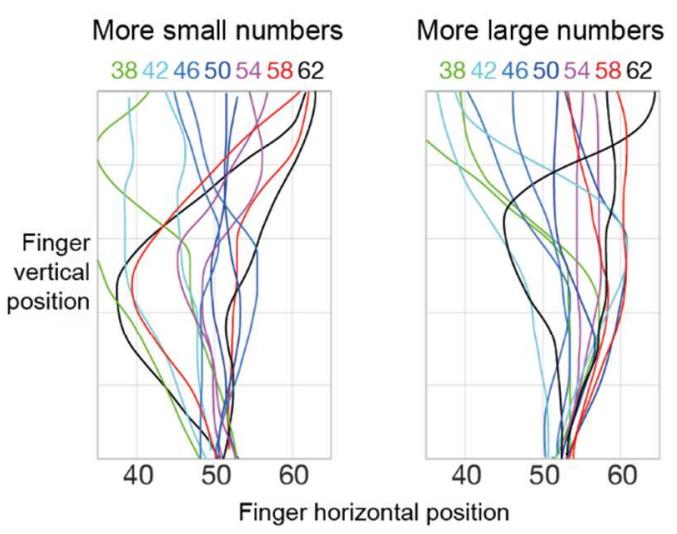

C Regression results
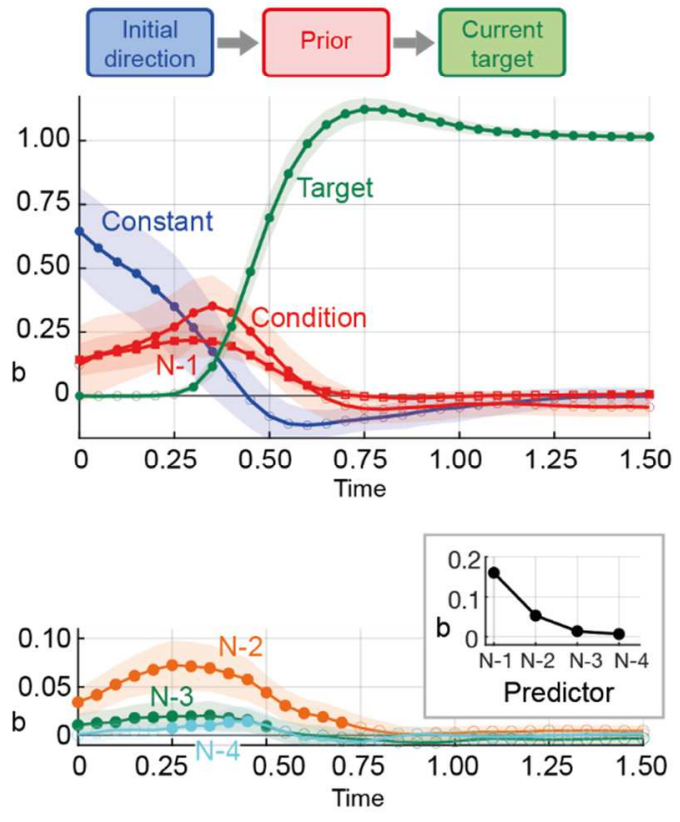

Fig. 2. Results of Experiment 1, which manipulated the distribution of target numbers in each block. (a) Average trajectory for each target number in each condition. The condition-induced bias is clearly visible in the early trajectory parts. (b) For some participants, the condition-induced bias can be observed even in single trials. (c) Results of regressing implied endpoints against the trial's target number, the 4 previous target numbers, and the average target value within each condition. The perparticipant regression $b$ values were averaged for each predictor and time point, and plotted as a function of time (full dot $=\mathrm{t}$-test significantly higher than 0 ). Shaded areas show $95 \%$ confidence intervals. $b$ [constant] (the regression intercept) is divided by 50 , so $b=1.0$ corresponds with the middle of the line. The colors in the top panel denote how the effects can be grouped into three sequential stages: [a] instruction-driven (blue), [b] prior-driven (red) and [c] target-driven (green). The inset in the bottom panel shows the mean $b$ value over the interval $0-600$ ms for each of the predictors $N-1, N-2$, $\mathrm{N}-3$, and $\mathrm{N}-4$ (four recent targets). Time $=0$ is the target onset. 
To completely rule out this recent-targets interpretation, we ran a new regression analysis, which was identical with the previous analysis except that the Condition predictor was replaced by the average of several trials back. The 4 recent trials were already included in the model as separate predictors, so the new trial-average predictor contained trials $\mathrm{N}-5$ to $\mathrm{N}-15$. If the Condition effect in the original regression was an artefact of recent trials, the trial-average predictor in the new regression should be a better predictor of finger movement, i.e., to have larger regression coefficients than those of the Condition predictor. The results, however, were completely the opposite: the trial-average predictor had significantly lower regression coefficients than those of the original Condition predictor (in each time point from 50 to $500 \mathrm{~ms}$, paired $\mathrm{t}(17)>2.47$, one-tailed $p<.02$ ). This difference was most evident in the time point when both predictors peaked, $350 \mathrm{~ms}$ (mean $\mathrm{b}$ [average of $\mathrm{N}-5$ to $\mathrm{N}-15$ ] $=0.12$ versus mean $\mathrm{b}$ [Condition $]=0.35$, paired $\mathrm{t}(17)=5.67$, one-tailed $p<.001)$. This superiority of the Condition predictor over the recent-trials-average predictor was observed also when the recent-trialsaverage predictor was set to consider only the average of trials $\mathrm{N}-5$ to $\mathrm{N}-10$, or the average of $\mathrm{N}-1$ to $\mathrm{N}-10$. The Condition effect therefore reflects a genuine effect of a prior based on the block-specific distribution of targets.

On top of the Condition effect, the finger was additionally affected by the target of the previous trial $(\mathrm{N}-1)$ and, to a smaller extent, by the targets of several trials farther back (bottom panel in Fig. 2c). The Condition and recent-trials effects rose and declined in similar time windows, suggesting that they resulted from a single cognitive process - the representation of a prior. Thus, the prior was not solely affected by the long-term distribution of targets, but also leaned towards the targets presented recently. The recent-targets effect decreased, roughly exponentially, for trials farther back (Fig. 2c inset), in agreement with the hypothesis that the prior is learned from accumulation of evidence over trials, with some forgetting of older information (Dotan \& Dehaene, 2016).

This pattern of results agrees with the three-stage process described in the Introduction: pointing to a default initial direction (blue); pointing according to the per-condition distribution of target numbers, i.e., according to a prior (red); and pointing to the estimated location of the target number (green). These three stages affected the finger sequentially: the effect of each stage started declining around the same time as the next effect started ascending.

\subsection{Experiment 2: manipulating the finger initial direction}

In Experiment 2, the distribution of targets was flat in all three blocks, but in each block the participants were instructed to move the finger in a different initial direction. Visual inspection of the average trajectories shows that the participants complied with this instruction (Fig. 3a). Single-trial trajectories suggested that, as predicted, in an intermediate stage, participants returned to pointing towards the middle of the number line, which is the direction implied by the prior in this experiment, even when this movement transiently carried the finger away from the target (Fig. 3b). For instance, when the target number was close to zero, and the instruction was to initially point towards 0 , the trajectory was not an optimal straight line, but often deviated transiently towards the middle of the line. 

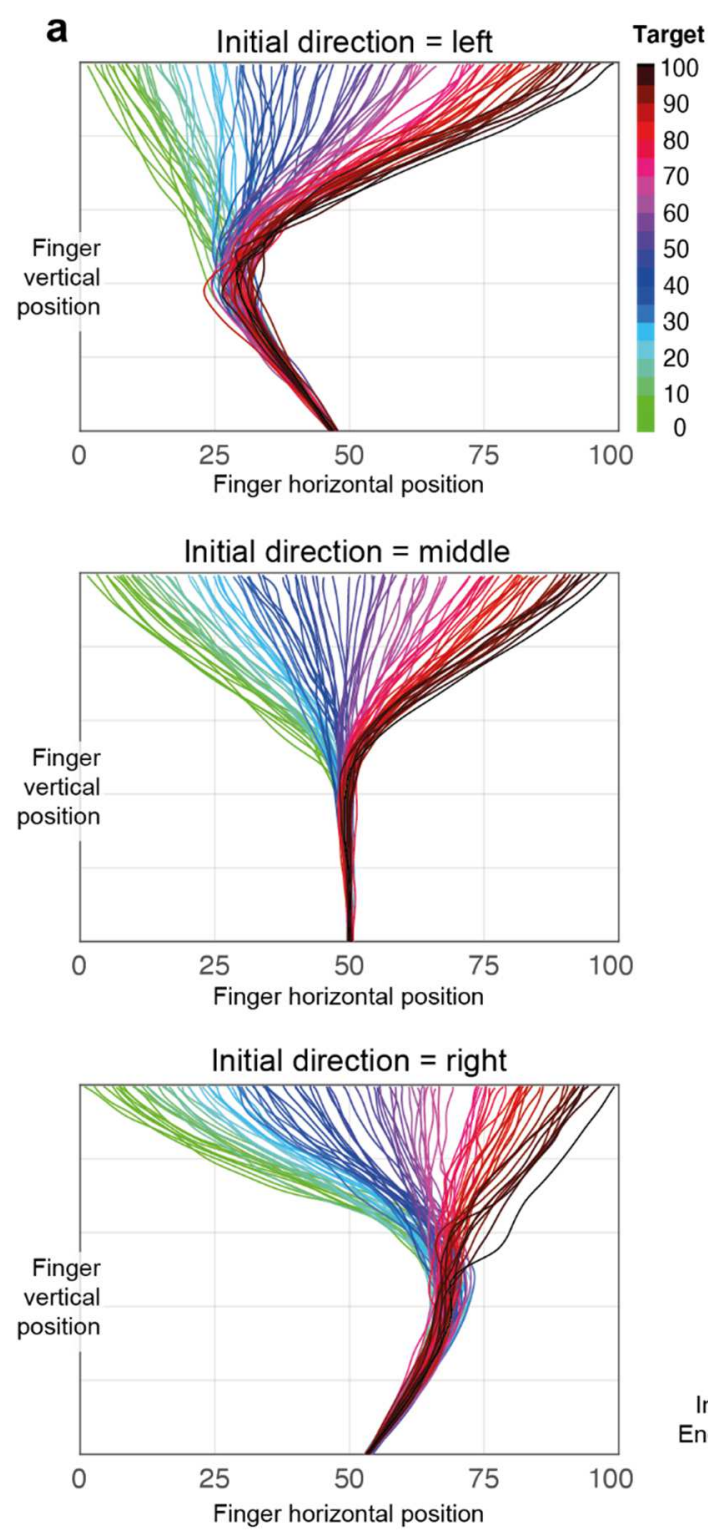

b Sample raw trajectories of one participant Initial direction $=$ left $\quad$ Initial direction $=$ right

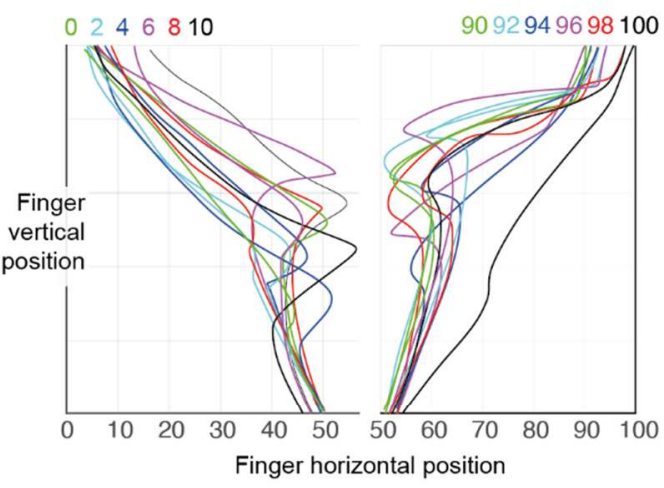

C Regression results
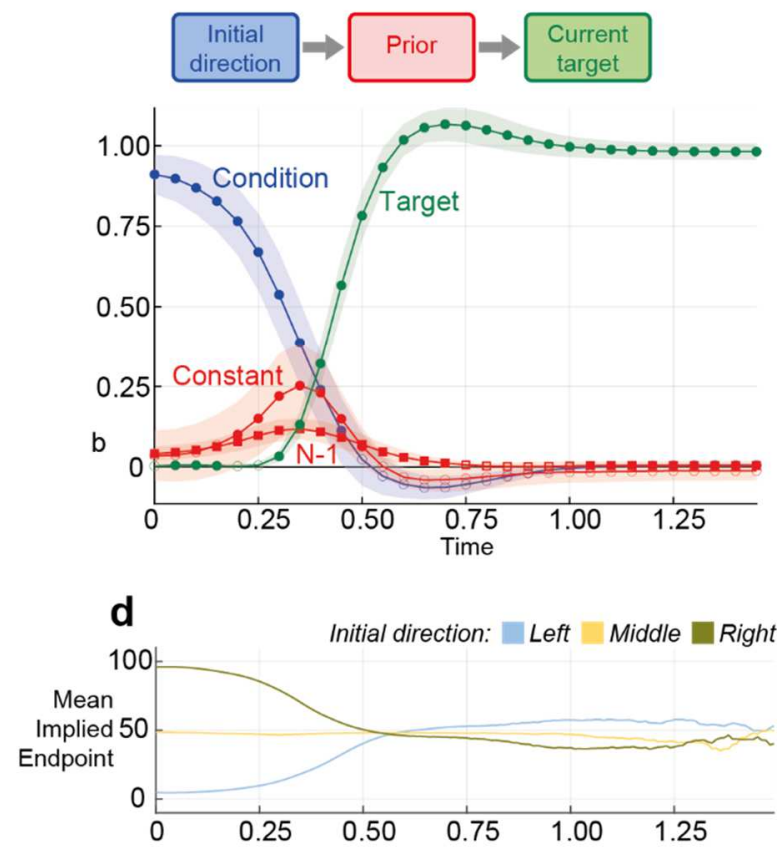

Fig. 3. Results of Experiment 2, which manipulated the initial direction of the finger in each block. (a) Average trajectory for each target number in each condition. Clearly, the participants complied with the instructed initial direction. (b) Single-trial trajectories: some participants transiently pointed towards the middle of the line (the location of the targets' average), even when this drove the finger away from the target number. (c) Time course of the effects (same plot type as Fig. 2c). Here the predictors were the target number of the present and previous trials $(\mathrm{N}-1)$ and the per-condition initial direction $(0,50$, or 100). The order of effects is the same as in Experiment 1 ( $a=$ instruction, $b=$ prior, $c=$ target), but here they are reflected by different predictors. (d) Average implied endpoint for condition in each time point. The initial average direction is different for each block, but about $200 \mathrm{~ms}$ into the trial it start converging into a common direction - the middle of the number line (target $=50$ ). In the regression curves (panel c), this convergence is reflected by the increase in the Constant effect, which also starts around $200 \mathrm{~ms}$. 
To quantify this pattern, trajectories were submitted to the regression analysis similar to Experiment 1: for each participant and for each time point in the trial, the implied endpoint was regressed against the Condition (the initial direction), the target number, and the target of the previous trial $\mathrm{N}-1$. Trials further back $(\mathrm{N}-2, \mathrm{~N}-3, \ldots)$ were not added as predictors because unlike Experiment 1, here they did not correlate with the Condition factor, so there was no need to control for them (but similar results were obtained when they were added). As in Experiment 1, three successive effects were observed (Fig. 3c). In the early trajectory parts (blue), the finger direction was dominated by the instructed initial direction (left/right/middle). This effect quickly declined, and completely disappeared by $550 \mathrm{~ms}$. In parallel, starting from 200 ms, the finger started moving towards a fixed direction (Constant effect, red color) - the middle of the number line (Fig. 3d) - and was additionally affected by the previous trial (N-1). As in Experiment 1, the two predictors of this middle stage unfolded with a similar time course, suggesting that they originated in a single cognitive process, the representation of the prior. Their peak was at $350 \mathrm{~ms}$, and then they declined as the finger direction became dominated by the target number (green).

This temporal organization again suggests the same three consecutive stages of pointing, first according to the initial direction (blue); then according to a prior, which in this experiment is a constant direction with an additional effect of the previous trial (red); and finally to the estimated location of the target number (green).

The results above indicate that the prior had an effect during an intermediate time window when examined over all trials, i.e., on average. We also examined the specific prediction that, on the particular trials in which the initial direction pointed straight at the target, the finger transiently deviated away from the target to accommodate the prior (Fig. 3b). In such situations, counter-intuitively, the trajectory should be less straight and more winding than when the initial direction points away from the target. To examine this prediction, we focused on the trials in which the target was close to either end of the number line (0-20 and 80-100; similar results were obtained when we avoided the single-digit targets and their mirrors, and considered only 10-20 and 80-90). We compared trials in which the initial direction and the target were congruent (initial $=$ right, target $\geq 80$; or initial $=$ left, target $\leq 20$ ) versus incongruent trials. To index whether a trajectory had many fluctuations we computed, for each trial, the number of bends (Dotan et al., 2019). A bend was defined as a trajectory section of at least $100 \mathrm{~ms}$ during which the finger continuously changed its direction in either clockwise or counter-clockwise manner. For this computation, we ignored the first $200 \mathrm{~ms}$ of each trial, to avoid random movements before the target was processed reliably, and the top $10 \%$ y coordinates of each trial, to avoid bends resulting from approach-to-numberline strategies. We also excluded very small bends, in which the finger direction changed by less than $5^{\circ}$ from the start to the end of the bend. To compare the two conditions statistically, we entered the per-trial number of bends as the dependent variable in a linear mixed model, with the participant as a random factor and with Initial-Target Congruency (congruent, incongruent) as a within-subject factor. We used a likelihood ratio test to compare this model versus a model in which the Congruency factor was 
removed. In accord with our prediction, the average number of bends in the congruent trials (2.51) was significantly higher than in the incongruent trials $\left(2.16 ; \chi^{2}(1)=247.1, p<.001\right)$. The number of bends in the above trials, in which the initial direction was congruent with the target but not with the prior, was also significantly higher than the number of bends in trials in which the initial direction was congruent both with the target and with the prior (initial direction = middle, target $=40-60$; average number of bends $=2.37, \chi^{2}(1)=35.8, p<.001$ ).

\section{Discussion}

In two different experiments, finger movement during decision making could be decomposed into three successive stages, with the same stages observed in both experiments: (a) Initially, the finger pointed to the instructed direction - either fixed (Experiment 1) or variable across blocks (Experiment 2). (b) Almost immediately, the finger started deviating towards the mean target of the experimental block. This effect started building up right from the start of the trial, and quickly reached significance. In Experiment 1, the target distribution was condition-dependent, so we observed a Condition effect in the regression. In Experiment 2, the target distribution was flat in all conditions, so the regressions showed a contribution of the constant term, as the average pointing direction started converging towards the midline. At this stage the finger was also influenced by the targets presented on recent trials, with a roughlyexponential decay as a function of temporal distance. This is precisely the pattern predicted for a stage in which the finger points according to a prior. (c) Finally, from $\sim 350 \mathrm{~ms}$ on, the finger started deviating towards the target number of the present trial.

Importantly, different experimental manipulations affected different processing stages. In Experiment 1, manipulating the distribution of target numbers affected stage $b$ but neither stage a nor stage c. In Experiment 2, manipulating the instructed initial direction affected stage a but neither $\mathrm{b}$ nor $\mathrm{c}$. This double dissociation indicates that the three stages of finger movement reflect three distinct cognitive processes.

The first stage (pointing by task instructions) and the third stage (pointing to target) are trivial: they simply reflect that the participants complied with the task instructions. Arguably, the first stage should perhaps not even be considered as decision-making. However, the existence of an additional interim stage is interesting, because the task did not require the participants to point according to the experimental block's average. The fact that they did supports the notion that for a short period, before the target was processed, the finger movement was affected by a prior, and only then the prior was overridden by the evidence accumulated in the trial.

An important result of Experiment 2 was that participants reverted to the prior even when this behavior transiently led their finger away from the target (Fig. 3b). Had we ignored the effect of prior, such behavior could seem suboptimal and even surprising. However, the notion of prior can easily explain why this is actually an efficient strategy on average: before the target information becomes available, the only basis for predicting the target location is the prior. Pointing according to this prior minimizes the average distance to the location of the upcoming target. As such, it also minimizes the average size of the corrective movement that will be later 
required to redirect to the target. More precisely, pointing towards the prior's mean minimizes the average quadratic error, while pointing towards its median minimizes the average absolute distance. The present data cannot distinguish between these possibilities, but subtle manipulations of the shapes of the target distributions might be able to address this point in the future.

Interestingly, during stage $b$, the finger direction was jointly affected by the average distribution of targets and by the targets presented on recent trials. The size of the recent-trials effect decreased more or less exponentially for trials farther back, replicating our previous findings (Dotan \& Dehaene, 2016). Importantly, we showed that these two effects (the targetdistribution effect and the recent-trials effect) are dissociable. Still, the two effects unfolded in almost perfect temporal alignment. This support the hypothesis, derived from our previous idealobserver model (Dotan \& Dehaene, 2016), that both effects originate in a single cognitive mechanism: the knowledge of the prior, which is based on the long-term distribution of observed targets, with an exponentially decreasing influence of recent targets. Previous mathematical modeling showed how such an exponential pattern arises when the prior gets updated by each new target number, with older targets being gradually forgotten (Abrahamyan et al., 2016; Cicchini et al., 2014; Dotan \& Dehaene, 2016).

Several alternative interpretations of the results can be ruled out. First, the findings cannot be explained under the assumption that finger movements are launched in a ballistic manner following a single decision per trial, because we clearly identified successive direction changes, sometimes observed even in single trials (Fig. 2b, 3b). Rather, the finger seems to be driven by the progressive accumulation of evidence (either continuously or in several discrete stages), resulting in changes in the finger direction in mid-trial, as observed in previous studies (Buc Calderon, Verguts, \& Gevers, 2015; Dotan et al., 2018; Pinheiro Chagas et al., 2017; see reviews in Dotan et al., 2019; Gallivan, Chapman, Wolpert, \& Flanagan, 2018).

Second, the effects that precede the target number (Constant, Condition, N-1) cannot be ascribed to a default behavior or a general aiming preference. Such default behavior should be observed right from the beginning of the trial, whereas in both experiments we observed some pre-target effects building up after the trial started, and peaking at $\sim 350 \mathrm{~ms}$. Importantly, these pre-target effects did not correspond to a fixed default behavior, but adapted flexibly to changes in the long-term prior and the history of recent targets.

Third, as noted above, the perfect alignment of the middle-stage effects - the target distribution effect and the recent-targets effect (red curves) - suggests that they have a single cognitive origin. Models that attribute these effects to separate mechanisms - e.g., explaining the distribution effect as an overt strategy, or the recent-trial effect as a motor-level perseveration cannot explain this parallelism.

Fourth, our findings cannot be explained by an averaging of several competing motor plans at the motor-control level. Recent studies indicate that such averaging does take place in some scenarios, including in point-to-target tasks: even before the trial-specific target is known, the motor system can prepare several movement plans towards potential target locations, and move 
the finger according to the average of these motor plans (Chapman et al., 2010a; Cisek, 2012; Gallivan et al., 2015, 2017). The resulting pattern resembles our data: the finger first aims towards the average location of the potential targets, and then redirects towards the trial-specific target location (Chapman et al., 2010a). The averaging of candidate target locations can even be probabilistic, at least to some extent. For example, if the participants are informed not only about the potential target locations but also that one location is more likely than the others, the trajectory slightly deviates towards that location (Hudson et al., 2007). However, our data is also different from those studies in two critical respects, indicating that our findings cannot be explained as motor-level averaging. First, the motor-control system can simultaneously represent only up to $\sim 4$ different motor plans to four different targets (Gallivan et al., 2011) - presumably, a working-memory limitation. This limit of four targets does not suffice to account for our data: each block in our experiments contained 101 different target numbers, and in both experiments, during the intermediate stage the finger pointed according to the average of the distribution of these 101 numbers. Crucially, we ruled out the possibility that the pointing-by-average stage in our data can be interpreted as pointing according to the average of the four recent trials. Second, in the studies that showed averaging of motor plans (Chapman et al., 2010a, 2010b; Gallivan et al., 2015, 2017), all potential target locations were shown in the beginning in each trial, so the participant had time to process the trial-specific potential targets and create the corresponding motor plans. In contrast, in our experiments, only one target was shown per trial, and the effect of prior was observed starting from very early time points in the trial (50 ms in Experiment 1$)-$ presumably, before there was enough time to process the target, reach a decision and propagate it to the motor system. Thus, the intermediate stage of pointing towards the experimental block's average could only rely on the participant's pre-trial knowledge about the experimental block's expected average target - essentially, some form of a prior. Note, however, that our findings cannot arbitrate whether this prior is a part of the decision stage, as we hypothesized, or of the motor-control processes (it was already proposed that an anticipated movement may have an effect within the motor control processes, Marinovic et al., 2017).

Methodologically, the present study confirms that finger tracking can shed light on the temporal dynamics of cognitive tasks (Dotan \& Dehaene, 2016; Dotan et al., 2018; Finkbeiner et al., 2014; Freeman et al., 2011; Friedman et al., 2013; Pinheiro-Chagas et al., 2017; Song \& Nakayama, 2008). Even more importantly for the present topic, we propose that the paradigm we introduced here may prove extremely useful to study decision making. Our study showed how the number-to-position task, which is typically used to study numerical cognition, can serve - in conjunction with finger tracking - to study decision making, in particular the representation of priors. This paradigm offers two major advantages. First, the number-to-position task has multiple response alternatives, whereas most other decision-making tasks have only two response alternatives. The large array of responses could be used to create complex patterns of target distributions and examine how they affect the prior. For example, an interesting question is whether the brain stores prior distributions in a detailed manner, e.g., one likelihood scalar value for each of the possible responses, or in a summarized manner, e.g., mean and standard deviation 
(Meyniel et al., 2015). Our paradigm may address this question by examining whether a bimodal distribution of targets (i.e., many large and small target numbers, with fewer mid-range numbers) yields a "bimodal prior". A possible prediction of such bimodal prior is that many trials will have a rightward or leftward bias, whereas only few trials will have no bias (i.e., will start by pointing to the middle of the number line). A second advantage of the number-to-position task is that it involves semantic access (to the quantity represented by the number), whereas most other tasks involve only perceptual decision making. At the same time, the number-to-position task is still simple enough to allow for accurate modeling (Cicchini et al., 2014; Dotan \& Dehaene, 2016).

Our data revealed that within a trial, before finger behavior reflected the target, it was governed by a prior. The exact organization of this prior, however, is yet unknown. In the future, the use of multiple-response paradigms such as the number-to-position task may be useful to investigate the organization of prior distribution, and to examine more accurately how the brain manipulates distributions and whether it operates, to a first approximation, as a Bayesian machine.

\section{References}

Abrahamyan, A., Silva, L. L., Dakin, S. C., Carandini, M., \& Gardner, J. L. (2016). Adaptable history biases in human perceptual decisions. Proceedings of the National Academy of Sciences, 113(25), E3548-E3557. https://doi.org/10.1073/pnas.1518786113

Al Roumi, F., Dotan, D., Yang, T., Wang, L., \& Dehaene, S. (2019). Acquisition and processing of an artificial mini-language combining semantic and syntactic elements. Cognition, 185, 49-61. https://doi.org/10.1016/j.cognition.2018.11.006

Barthelmé, S., \& Mamassian, P. (2010). Flexible mechanisms underlie the evaluation of visual confidence. Proceedings of the National Academy of Sciences, 107(48), 20834-20839. https://doi.org/10.1073/pnas.1007704107

Berteletti, I., Lucangeli, D., Piazza, M., Dehaene, S., \& Zorzi, M. (2010). Numerical estimation in preschoolers. Developmental Psychology, 46(2), 545-551. https://doi.org/10.1037/a0017887

Buc Calderon, C., Verguts, T., \& Gevers, W. (2015). Losing the boundary: Cognition biases action well after action selection. Journal of Experimental Psychology: General, 144(4), 737-743. https://doi.org/10.1037/xge0000087

Chapman, C. S., Gallivan, J. P., Wood, D. K., Milne, J. L., Culham, J. C., \& Goodale, M. A. (2010a). Reaching for the unknown: Multiple target encoding and real-time decision-making in a rapid reach task. Cognition, 116(2), 168-176. https://doi.org/https://doi.org/10.1016/j.cognition.2010.04.008

Chapman, C. S., Gallivan, J. P., Wood, D. K., Milne, J. L., Culham, J. C., \& Goodale, M. A. (2010b). Short-term motor plasticity revealed in a visuomotor decision-making task. Behavioural Brain Research, 214(1), 130-134. https://doi.org/https://doi.org/10.1016/j.bbr.2010.05.012

Cicchini, G. M., Anobile, G., \& Burr, D. C. (2014). Compressive mapping of number to space reflects dynamic encoding mechanisms, not static logarithmic transform. Proceedings of the National Academy of Sciences of the United States of America, 111(21), 7867-7872. https://doi.org/10.1073/pnas.1402785111

Cisek, P. (2012). Making decisions through a distributed consensus. Current Opinion in Neurobiology, 22(6), 927-936. https://doi.org/https://doi.org/10.1016/j.conb.2012.05.007

Dehaene, S. (1997). The number sense: How the mind creates mathematics. Oxford University Press. 
Dehaene, S., Izard, V., Spelke, E., \& Pica, P. (2008). Log or linear? Distinct intuitions of the number scale in Western and Amazonian indigene cultures. Science, 320, 1217-1220. https://doi.org/10.1126/science. 1156540

Dotan, D., \& Dehaene, S. (2013). How do we convert a number into a finger trajectory? Cognition, 129(3), 512-529. https://doi.org/10.1016/j.cognition.2013.07.007

Dotan, D., \& Dehaene, S. (2016). On the origins of logarithmic number-to-position mapping. Psychological Review, 123(6), 637-666. https://doi.org/10.1037/rev0000038

Dotan, D., \& Dehaene, S. (2020). Parallel and serial processes in number-to-quantity conversion. Cognition, 204, 104387. https://doi.org/10.1016/j.cognition.2020.104387

Dotan, D., Meyniel, F., \& Dehaene, S. (2018). On-line confidence monitoring during decision making. Cognition, 171, 112-121. https://doi.org/10.1016/j.cognition.2017.11.001

Dotan, D., Pinheiro-Chagas, P., Al Roumi, F., \& Dehaene, S. (2019). Track it to crack it: Dissecting processing stages with finger tracking. Trends in Cognitive Sciences, 23(12), 1058-1070. https://doi.org/10.1016/j.tics.2019.10.002

Erb, C. D., Moher, J., Sobel, D. M., \& Song, J. H. (2016). Reach tracking reveals dissociable processes underlying cognitive control. Cognition, 152, 114-126. https://doi.org/10.1016/j.cognition.2016.03.015

Fernandes, H. L., Stevenson, I. H., Vilares, I., \& Kording, K. P. (2014). The generalization of prior uncertainty during reaching. The Journal of Neuroscience, 34(34), 11470 LP - 11484. https://doi.org/10.1523/JNEUROSCI.3882-13.2014

Finkbeiner, M., Coltheart, M., \& Coltheart, V. (2014). Pointing the way to new constraints on the dynamical claims of computational models. Journal of Experimental Psychology: Human Perception and Performance, 40(1), 172-185. https://doi.org/10.1037/a0033169

Finkbeiner, M., \& Friedman, J. (2011). The flexibility of nonconsciously deployed cognitive processes: Evidence from masked congruence priming. PLOS ONE, 6(2), e17095. https://doi.org/10.1371/journal.pone.0017095

Freeman, J. B., Dale, R., \& Farmer, T. A. (2011). Hand in motion reveals mind in motion. Frontiers in Psychology, 2. https://doi.org/10.3389/fpsyg.2011.00059

Friedman, J., Brown, S., \& Finkbeiner, M. (2013). Linking cognitive and reaching trajectories via intermittent movement control. Journal of Mathematical Psychology, 57, 140-151. https://doi.org/10.1016/j.jmp.2013.06.005

Gallivan, J. P., Barton, K. S., Chapman, C. S., Wolpert, D. M., \& Randall Flanagan, J. (2015). Action plan co-optimization reveals the parallel encoding of competing reach movements. Nature Communications, 6, 7428. https://doi.org/10.1038/ncomms8428

Gallivan, J. P., Chapman, C. S., Wolpert, D. M., \& Flanagan, J. R. (2018). Decision-making in sensorimotor control. Nature Reviews Neuroscience, 19(9), 519-534. https://doi.org/10.1038/s41583018-0045-9

Gallivan, J. P., Chapman, C. S., Wood, D. K., Milne, J. L., Ansari, D., Culham, J. C., \& Goodale, M. A. (2011). One to four, and nothing more: Nonconscious parallel individuation of objects during action planning. Psychological Science, 22(6), 803-811. https://doi.org/10.1177/0956797611408733

Gallivan, J. P., Stewart, B. M., Baugh, L. A., Wolpert, D. M., \& Flanagan, J. R. (2017). Rapid automatic motor encoding of competing reach options. Cell Reports, 18(7), 1619-1626.

https://doi.org/https://doi.org/10.1016/j.celrep.2017.01.049

Glimcher, P. W. (2003). The neurobiology of visual-saccadic decision making. Annual Review of Neuroscience, 26(1), 133-179. https://doi.org/10.1146/annurev.neuro.26.010302.081134

Gold, J. I., \& Shadlen, M. N. (2002). Banburismus and the brain. Neuron, 36(2), 299-308. https://doi.org/10.1016/S0896-6273(02)00971-6 
Gold, J. I., \& Shadlen, M. N. (2007). The neural basis of decision making. Annual Review of Neuroscience, 30(1), 535-574. https://doi.org/10.1146/annurev.neuro.29.051605.113038

Hanks, T. D., Mazurek, M. E., Kiani, R., Hopp, E., \& Shadlen, M. N. (2011). Elapsed decision time affects the weighting of prior drobability in a perceptual decision task. Journal of Neuroscience, 31(17), 6339-6352. https://doi.org/10.1523/JNEUROSCI.5613-10.2011

Hudson, T. E., Maloney, L. T., \& Landy, M. S. (2007). Movement planning with probabilistic target information. Journal of Neurophysiology, 98(5), 3034-3046.

https://doi.org/10.1152/JN.00858.2007/ASSET/IMAGES/LARGE/Z9K0110785550006.JPEG

Kiani, R., Corthell, L., \& Shadlen, M. N. (2014). Choice certainty is informed by both evidence and decision time. Neuron, 84(6), 1329-1342. https://doi.org/10.1016/j.neuron.2014.12.015

Kiani, R., Hanks, T. D., \& Shadlen, M. N. (2008). Bounded integration in parietal cortex underlies decisions even when viewing duration is dictated by the environment. Journal of Neuroscience, 28(12), 3017-3029. https://doi.org/10.1523/JNEUROSCI.4761-07.2008

Kiani, R., \& Shadlen, M. N. (2009). Representation of confidence associated with a decision by neurons in the parietal cortex. Science, 324(5928), 759-764. https://doi.org/10.1126/science.1169405

Knill, D. C., \& Pouget, A. (2004). The Bayesian brain: the role of uncertainty in neural coding and computation. Trends in Neurosciences, 27(12), 712-719. https://doi.org/10.1016/j.tins.2004.10.007

Kording, K. P., \& Wolpert, D. M. (2004). Bayesian integration in sensorimotor learning. Nature, 427(6971), 244-247. https://doi.org/10.1038/nature02169

Lo, C. C., \& Wang, X. J. (2006). Cortico-basal ganglia circuit mechanism for a decision threshold in reaction time tasks. Nature Neuroscience, 9(7), 956-963. https://doi.org/10.1038/nn1722

Marinovic, W., Poh, E., de Rugy, A., \& Carroll, T. J. (2017). Action history influences subsequent movement via two distinct processes. ELife, 6, e26713. https://doi.org/10.7554/eLife.26713

Meyniel, F., Sigman, M., \& Mainen, Z. F. (2015). Confidence as Bayesian probability: From neural origins to behavior. Neuron, 88(1), 78-92. https://doi.org/10.1016/j.neuron.2015.09.039

O’Connell, R. G., Dockree, P. M., \& Kelly, S. P. (2012). A supramodal accumulation-to-bound signal that determines perceptual decisions in humans. Nature Neuroscience, 15(12), 1729-1735. http://dx.doi.org/10.1038/nn.3248

Pinheiro-Chagas, P., Dotan, D., Piazza, M., \& Dehaene, S. (2017). Finger tracking reveals the covert stages of mental arithmetic. Open Mind, 1(1), 30-41. https://doi.org/10.1162/OPMI_a_00003

Ratcliff, R., \& McKoon, G. (2007). The diffusion decision model: Theory and data for two-choice decision tasks. Neural Computation, 20(4), 873-922. https://doi.org/10.1162/neco.2008.12-06-420

Ratcliff, R., \& Rouder, J. N. (1998). Modeling Response Times for Two-Choice Decisions. Psychological Science, 9(5), 347-356. https://doi.org/10.1111/1467-9280.00067

Roitman, J., \& Shadlen, M. N. (2002). Response of neurons in the lateral intraparietal area during a combined visual discrimination reaction time task. The Journal of Neuroscience, 22(21), 9475-9489. http://www.jneurosci.org/content/22/21/9475.abstract

Santens, S., Goossens, S., \& Verguts, T. (2011). Distance in motion: response trajectories reveal the dynamics of number comparison. PLoS ONE, 6(9), Article ID e25429. https://doi.org/10.1371/journal.pone.0025429

Scherbaum, S., \& Kieslich, P. J. (2017). Stuck at the starting line: How the starting procedure influences mouse-tracking data. Behavior Research Methods. https://doi.org/10.3758/s13428-017-0977-4

Siegler, R. S., \& Opfer, J. E. (2003). The development of numerical estimation: Evidence for multiple representations of numerical quantity. Psychological Science, 14(3), 237-250.

https://doi.org/10.1111/1467-9280.02438 
Slusser, E., \& Barth, H. C. (2017). Intuitive proportion judgment in number-line estimation: Converging evidence from multiple tasks. Journal of Experimental Child Psychology, 162, 181-198. https://doi.org/https://doi.org/10.1016/j.jecp.2017.04.010

Smith, P. L., \& Ratcliff, R. (2004). Psychology and neurobiology of simple decisions. Trends in Neurosciences, 27(3), 161-168. https://doi.org/10.1016/j.tins.2004.01.006

Song, J. H., \& Nakayama, K. (2008). Numeric comparison in a visually-guided manual reaching task. Cognition, 106(2), 994-1003. https://doi.org/10.1016/j.cognition.2007.03.014

Spivey, M. J., Grosjean, M., \& Knoblich, G. (2005). Continuous attraction toward phonological competitors. Proceedings of the National Academy of Sciences of the United States of America, 102(29), 10393 LP - 10398. http://www.pnas.org/content/102/29/10393.abstract

Stillman, P. E., Shen, X., \& Ferguson, M. J. (2018). How mouse-tracking can advance social cognitive theory. Trends in Cognitive Sciences, 22(6), 531-543. https://doi.org/https://doi.org/10.1016/j.tics.2018.03.012

Stocker, A. A., \& Simoncelli, E. P. (2006). Noise characteristics and prior expectations in human visual speed perception. Nature Neuroscience, 9(4), 578-585. https://doi.org/10.1038/nn1669

Summerfield, C., \& de Lange, F. P. (2014). Expectation in perceptual decision making: Neural and computational mechanisms. Nature Reviews Neuroscience, 15(11), 745-756. http://dx.doi.org/10.1038/nrn3838

Tassinari, H., Hudson, T. E., \& Landy, M. S. (2006). Combining priors and noisy visual cues in a rapid pointing task. The Journal of Neuroscience, 26(40), 10154-10163. https://doi.org/10.1523/JNEUROSCI.2779-06.2006

Yang, T., \& Shadlen, M. N. (2007). Probabilistic reasoning by neurons. Nature, 447(7148), 1075-1080. https://doi.org/10.1038/nature05852 